\title{
ON THE UNIMODALITY AND THE BELL-SHAPE OF NONCENTRAL DISTRIBUTIONS*
}

\author{
ANDREA VAN AUBEL ${ }^{\dagger}$ AND WOLFGANG GAWRONSKI ${ }^{\ddagger}$
}

\begin{abstract}
Various analytic properties of the noncentral $\chi_{n}^{2}(\lambda), F_{n_{1}, n_{2}}(\lambda)$, and student's $t_{m}(\lambda)$ distribution are studied. Supplementing earlier results of Das Gupta, Sarkar and Minko, Petunin, who showed the strong and the strict unimodality of the $\chi_{n}^{2}(\lambda)$ distribution respectively, in this paper it is proved that strict unimodality for $F_{n_{1}, n_{2}}(\lambda)$ and $t_{m}(\lambda)$ holds as well. For all three distributions the strict unimodality is obtained as a consequence of a sharper result saying that each of the corresponding densities is bell-shaped. Moreover in any case the unique mode is a strictly increasing function with respect to the noncentrality parameter $\lambda$.
\end{abstract}

0. Introduction and summary. Classical statistical tests, which are based on normally distributed observations, naturally lead to the noncentral $\chi_{n}^{2}(\lambda), F_{n_{1}, n_{2}}(\lambda)$, and student's $t_{m}(\lambda)$ distributions. Here and throughout we adhere to the terminology of Johnson, Kotz, Balakrishnan (1995) where $\lambda$ is the noncentrality parameter and $n, n_{1}, n_{2}, m>0$ denote the various degrees of freedom. In view of their statistical meaning usually $n, n_{1}, n_{2}, m$ are restricted to positive integers. However in this paper it is convenient to consider the degrees of freedom as positive variables rather than as discrete parameters. It is well-known that the distributions $\chi_{n}^{2}(\lambda)$ and $F_{n_{1}, n_{2}}(\lambda), \lambda \geq 0$, both are supported on the positive half axis and possess densities being analytic functions on $(0, \infty)$, whereas the $t_{m}(\lambda)$ distribution, $\lambda \in \mathbb{R}$, is supported on the whole real line with an analytic density on $\mathbb{R}$. For a collection of explicit formulae we refer to Section 1. It is the purpose of this paper to prove a series of analytic properties for the aforementioned probability distributions.

We begin with unimodality and remind some basic definitions. Suppose that $F$ is a probability distribution function on the real axis. Then $F$ is called unimodal with mode $M$ if $F$ is convex on $(-\infty, M)$ and concave on $(M, \infty)$ (Dharmadhikari, Joag-dev (1988)). Further let $a:=\inf \{x \in \mathbb{R} \mid F(x)>0\}$ and $b:=\sup \{x \in$ $\mathbb{R} \mid F(x)<1\}$. We call $F$ strictly unimodal with mode $M \in(a, b)$, if $F$ is absolutely continuous on $(-\infty, M) \cup(M, \infty)$ with density $f=F^{\prime}$ such that $f$ is strictly increasing on $(a, M)$ and strictly decreasing on $(M, b)$ (Sato, Yamazato (1978)). Obviously strict unimodality implies "simple" unimodality and the uniqueness of the mode. Apparently when dealing with convolutions the concept of strong unimodality is important (Ibragimov (1956)). Here $F$ is called strongly unimodal if the convolution $F * G$ again is unimodal for all unimodal distribution functions $G$. Consequently every strongly unimodal distribution a fortiori is unimodal.

Since all noncentral distributions under consideration possess an analytic density, $p$ say, on their intervals of support, showing strict unimodality amounts to proving that the equation $p^{\prime}(x)=0$ has exactly one solution $x=M \in(0, \infty)$ ( or $\mathbb{R}$ ) which then is the corresponding mode. In the central case, i.e. $\lambda=0$, for all three distributions the densities are elementary functions and a unimodality proof reduces to an easy exercise of beginner's calculus. However, if $\lambda \neq 0$, then for all densities only representations in terms of infinite series, parameter integrals, and higher transcendental functions are known. This lack of simplicity, as known from other important probability distributions (Sato, Yamazato (1978), Yamazato (1978)),

${ }^{*}$ Received 10 December, 98; revised August 6, 99.

${ }^{\dagger}$ Bredowallee 11, D-53125 Bonn, Germany.

$\ddagger$ Abteilung Mathematik, Universität Trier, D-54286 Trier, Germany (gawron@uni-trier.de). 
in general causes some difficulties for proving unimodality. At various places in the literature the unimodality of the noncentral distributions $\chi_{n}^{2}(\lambda), F_{n_{1}, n_{2}}(\lambda), t_{m}(\lambda)$ is claimed or silently assumed which is supported by graphical plots obtained by numerical approximations (e.g. Johnson et al. (1995), Kühlmeyer (1970), Narula, Levy (1975)). But as far as the authors are aware the only rigorous unimodality proof exists for the noncentral $\chi_{n}^{2}(\lambda)$ distribution. Using the convolution structure of the $\chi_{n}^{2}(\lambda)$ distribution Das Gupta, Sarkar (1984) proved the log-concavity for the corresponding density which is equivalent to strong unimodality via the well-known criterion of Ibragimov (1956). Minko, Petunin (1988) showed the strict unimodality of the $\chi_{n}^{2}(\lambda)$ distribution provided $\lambda \geq 0, n>2$ or $\lambda>2, n=2(n \in \mathbb{N})$, by using intricate properties for quotients of Bessel functions.

We prove strict unimodality for all three types of noncentral distributions (Section 2) by verifying the stronger property that the corresponding densities are "bellshaped". The latter notion is intimately connected with the graph of the density of the normal distribution. To be precise we call a probability density $p$ bell-shaped of order $r \in \mathbb{N}$, if the support of $p$ is an open interval, $(a, b)$ say, and if for all positive integers $k \leq r$ the derivative $p^{(k)}$ exists and has precisely $k$ zeros on $(a, b)$ and all zeros are simple. If the latter property holds for every order $r$, then for short we say that $p$ is bell-shaped (Karlin (1968), p. 325). Thus a bell-shaped density of some order belongs to a strictly unimodal distribution. In view of the Rodrigues formula for Hermite polynomials $H_{k}$

$$
H_{k}(x)=(-1)^{k} e^{x^{2}}\left(\frac{d}{d x}\right)^{k} e^{-x^{2}}, \quad k \in \mathbb{N},
$$

(Szegö (1985), p. 106, (5.5.3)) and the fact that $H_{k}$ has only real zeros which are all simple we have a precise formulation that the density of the normal distribution is bell-shaped. The density of the central $\chi_{n}^{2}$ distribution is given by

$$
p_{n}(x)=\frac{1}{2} \frac{e^{-x / 2}}{\Gamma(n / 2)}\left(\frac{x}{2}\right)^{\frac{n}{2}-1}, \quad x>0,
$$

$n>0$. Now by the Rodrigues formula for the Laguerre polynomials $L_{k}^{(\alpha)}$ (Szegö (1985), p. 101)

$$
p_{n}^{(k)}(x)=\frac{k !}{2^{k}} p_{n}(x)\left(\frac{x}{2}\right)^{-k} L_{k}^{\left(\frac{n}{2}-k-1\right)}\left(\frac{x}{2}\right), \quad x>0,
$$

and, since the zeros of the orthogonal polynomials $L_{k}^{(\alpha)}, \alpha>-1$, all are simple and positive, we get the bell-shape of order $\left\lfloor\frac{n}{2}\right\rfloor$ for the central $\chi_{n}^{2}$ density, $n>2$. Here and throughout the paper for real $\xi$ the symbol $\lfloor\xi\rfloor$ denotes the integer which is uniquely defined by $\lfloor\xi\rfloor<\xi \leq\lfloor\xi\rfloor+1$. Similar statements are readily verified for the central $F_{n_{1}, n_{2}}$ and $t_{m}$ densities. In Section 2 we establish that the density of the noncentral $\chi_{n}^{2}(\lambda)$ distribution is bell-shaped of order $\left\lfloor\frac{n}{2}\right\rfloor$, when $n>2, \lambda \geq 0$, the density of the noncentral $F_{n_{1}, n_{2}}(\lambda)$ distribution is bell-shaped of order $\left\lfloor\frac{n_{1}}{2}\right\rfloor$, when $n_{1}>2, n_{2}>0, \lambda \geq 0$, and the density of the noncentral $t_{m}(\lambda)$ distribution is bell-shaped, when $m>0, \lambda \in \mathbb{R}$. Hence all these distributions are strictly unimodal. The underlying proofs are based on a unified approach using essentially the concept of total positivity and in particular certain variation diminishing transformations of the type

$$
\int_{0}^{\infty} K(x, y) f(y) d y
$$


where $K$ is a suitable "sign regular" kernel. Concerning the theory of total positivity we refer to the standard monograph of Karlin (1968). We have listed some of the relevant ingredients of this theory in the next section.

Another look at the graphs of the densities under consideration suggests monotonic dependence of various quantities with respect to $\lambda$ and the degrees of freedom (e.g. Johnson et al. (1995), Kühlmeyer (1970), Narula, Levy (1975)). In Section 3 for all cases we prove that the mode is a strictly increasing function of the noncentrality parameter. Furthermore we study monotonicity properties of the mode with respect to the degree of freedom in case of $\chi_{n}^{2}(\lambda)$ and $F_{n_{1}, n_{2}}(\lambda)$ and the variation of all densities with $\lambda$. The proofs again make use of the theory of totally positive kernels.

1. Auxiliary results. In this section we fix the precise terminology and collect some preliminary results most of which are known in the literature.

We start with the definition of the underlying noncentral distributions in terms of the corresponding densities (Johnson et al. (1995)). Throughout the paper we assume that the positive numbers $n, n_{1}, n_{2}, m$ (not necessarily integers) denote the degrees of freedom of the noncentral distributions and the real number $\lambda$ indicates the noncentrality parameter. The central $\chi_{n}^{2}$ distribution is defined through its density

$$
p_{n, 0}(x):=\frac{1}{2} \frac{e^{-x / 2}}{\Gamma(n / 2)}\left(\frac{x}{2}\right)^{\frac{n}{2}-1}, \quad x>0,
$$

and the noncentral $\chi_{n}^{2}(\lambda)$ distribution, $\lambda \geq 0$, is given by its density

$$
p_{n, \lambda}(x):=\sum_{j=0}^{\infty} \frac{e^{-\lambda / 2}}{j !}\left(\frac{\lambda}{2}\right)^{j} p_{n+2 j, 0}(x), \quad x>0 .
$$

The density of the central student's $t_{m}$ distribution is defined by

$$
h_{m, 0}(x):=\frac{1}{\sqrt{m}} \frac{\Gamma\left(\frac{m+1}{2}\right)}{\Gamma\left(\frac{m}{2}\right) \Gamma\left(\frac{1}{2}\right)}\left(1+\frac{x^{2}}{m}\right)^{-\frac{m+1}{2}}, \quad x \in \mathbb{R},
$$

whereas in the noncentral case the density of the $t_{m}(\lambda)$ distribution, $\lambda \in \mathbb{R}$, is given by

$$
h_{m, \lambda}(x):=\frac{1}{2^{\frac{m+1}{2}} \Gamma\left(\frac{m}{2}\right) \sqrt{m \pi}} \int_{0}^{\infty} t^{\frac{m-1}{2}} \exp \left(-\frac{t}{2}-\frac{1}{2}\left(x \sqrt{\frac{t}{m}}-\lambda\right)^{2}\right) d t, x \in \mathbb{R} .
$$

Putting

$$
\tilde{g}_{n_{1}, n_{2}, 0}(x):=\frac{\Gamma\left(\frac{n_{1}+n_{2}}{2}\right)}{\Gamma\left(\frac{n_{1}}{2}\right) \Gamma\left(\frac{n_{2}}{2}\right)} \frac{x^{\frac{n_{1}}{2}-1}}{(1+x)^{\frac{n_{1}+n_{2}}{2}}}, \quad x>0
$$

by

$$
g_{n_{1}, n_{2}, 0}(x):=\frac{n_{1}}{n_{2}} \tilde{g}_{n_{1}, n_{2}, 0}\left(\frac{n_{1}}{n_{2}} x\right), \quad x>0,
$$

we denote the density of the central $F_{n_{1}, n_{2}}$ distribution and further with

$$
\tilde{g}_{n_{1}, n_{2}, \lambda}(x):=\sum_{j=0}^{\infty} \frac{e^{-\lambda / 2}}{j !}\left(\frac{\lambda}{2}\right)^{j} \tilde{g}_{n_{1}+2 j, n_{2}, 0}(x), \quad x>0
$$


the density of the noncentral $F_{n_{1}, n_{2}}(\lambda)$ distribution, $\lambda \geq 0$, is defined through

$$
g_{n_{1}, n_{2}, \lambda}(x):=\frac{n_{1}}{n_{2}} \tilde{g}_{n_{1}, n_{2}, \lambda}\left(\frac{n_{1}}{n_{2}} x\right), \quad x>0 .
$$

Following the approach in the analysis of variance the $F_{n_{1}, n_{2}}(\lambda)$ distribution can be defined as that of the quotient of the quadratic forms $\frac{1}{n_{1}} \sum_{j=1}^{n_{1}} X_{1 j}^{2}$ and $\frac{1}{n_{2}} \sum_{j=1}^{n_{2}} X_{2 j}^{2}$, where $X_{11}, \ldots, X_{1 n_{1}}, X_{21}, \ldots, X_{2 n_{2}}$ are independent random variables, $X_{1 j}$ being normally distributed with expectation $\delta_{j}$ and variance $1, \lambda=\sum_{j=1}^{n_{1}} \delta_{j}^{2}$, and $X_{2 j}$ are standard normal variates. Omitting both factors $\frac{1}{n_{1}}$ and $\frac{1}{n_{2}}$ the corresponding quotient is distributed according to the density (1.7). The associated distribution is called rescaled $\tilde{F}_{n_{1}, n_{2}}(\lambda)$ distribution which for some instances is easier to handle than the $F_{n_{1}, n_{2}}(\lambda)$ distribution (e.g. Das Gupta, Perlman (1974), Sarkar (1983)). In any case the central distribution is obtained from the noncentral one by putting $\lambda=0$. Next we introduce the modified Bessel function of the first kind and order $\nu \in \mathbb{R}$ (e.g. Watson (1966), p. 77) given by the expansion

$$
I_{\nu}(z)=\sum_{k=0}^{\infty} \frac{1}{\Gamma(k+\nu+1) k !}\left(\frac{z}{2}\right)^{2 k+\nu}
$$

which throughout we use for real and positive $z$ only. For further reference we record the following representation of $I_{\nu}$ as an infinite product involving the zeros $j_{\nu, k}$ of the Bessel function $J_{\nu}$ (Watson (1966), pp. 77, 482, 498, 502) in

LEMMA 1.1. For $\nu>-1$ and $z>0$ we have

$$
I_{\nu}(z)=\frac{(z / 2)^{\nu}}{\Gamma(\nu+1)} \prod_{k=1}^{\infty}\left(1+\frac{z^{2}}{j_{\nu, k}^{2}}\right)
$$

where $0<j_{\nu, 1}<j_{\nu, 2}<\ldots$ and

$$
\sum_{k=1}^{\infty} \frac{1}{j_{\nu, k}^{2}}=\frac{1}{4(\nu+1)}
$$

As consequences from the definitions above we infer some known representation formulae (see Johnson et al. (1995), chapters 29, 30) in

LEMMA 1.2. With the notations above for $x>0$ we have

$$
\begin{aligned}
& p_{n, \lambda}(x)=\frac{1}{2} e^{-(\lambda+x) / 2}\left(\frac{x}{\lambda}\right)^{\frac{n-2}{4}} I_{\frac{n-2}{2}}(\sqrt{\lambda x}) \\
& p_{n, \lambda}(x)=\int_{0}^{\infty} p_{m, \delta}(x-y) p_{n-m, \lambda-\delta}(y) d y
\end{aligned}
$$

where $0<m<n, 0 \leq \delta \leq \lambda$,

$$
\begin{gathered}
\tilde{g}_{n_{1}, n_{2}, \lambda}(x)=\int_{0}^{\infty} p_{n_{1}, \lambda}(x y) p_{n_{2}, 0}(y) y d y \\
g_{n_{1}, n_{2}, \lambda}(x)=\frac{n_{1}}{n_{2}} \int_{0}^{\infty} p_{n_{1}, \lambda}\left(\frac{n_{1}}{n_{2}} x y\right) p_{n_{2}, 0}(y) y d y .
\end{gathered}
$$


Our next auxiliary results use some special properties of functions of two real variables. More precisely, they are based on various concepts such as total positivity $(\mathrm{TP})$, sign regularity (SR), reverse regularity $(\mathrm{RR})$, Polya frequency functions (PF) and related ones. We take the definitions of these notions and standard results from Karlin's monograph (1968) which we choose as basic reference work for some relevant analytic properties of the noncentral densities $p_{n, \lambda}, g_{n_{1}, n_{2}, \lambda}, h_{m, \lambda}$ as well. In particular we make frequent use of extended sign regular functions of order $r \in \mathbb{N}\left(E S R_{r}\right)$. These are real valued functions $K \in C_{r-1}(X \times Y)(X, Y \subset \mathbb{R}$ being open intervals) such that for each $m=1, \ldots, r$ the sign

$$
\varepsilon_{m}(K):=\operatorname{sign} \operatorname{det}\left(\frac{\partial^{i+j-2}}{\partial x^{i-1} \partial y^{j-1}} K(x, y)\right)_{i, j=1}^{m}
$$

is constant and different from zero on $X \times Y$; that is $\left(\varepsilon_{m}(K)\right)_{m=1}^{r}$ is a finite sequence of plus and minus ones. In case $\varepsilon_{m}(K)=1, m=1, \ldots, r, K(x, y)$ is called extended totally positive of order $r\left(\mathrm{ETP}_{r}\right)$ with respect to $(x, y)$ on $X \times Y$ and if $\varepsilon_{m}(K)=(-1)^{m(m-1) / 2}, m=1, \ldots, r$, then the attribute extended reverse regular of order $r\left(\mathrm{ERR}_{r}\right)$ is used. Further the notations $\mathrm{ESR}_{\infty}, \mathrm{ETP}_{\infty}, \mathrm{ERR}_{\infty}$ are employed, when the defining property holds for any $r \in \mathbb{N}$. Many interesting kernels $K(x, y)$ are generated by simple kernels. Therefore we collect some composition laws which are given in Karlin (1968) directly or can easily be derived via standard techniques from total positivity (Karlin (1968), pp. 99, 157).

Lemma 1.3. Suppose that $X, Y, Z, U, V \subset \mathbb{R}$ are open intervals, $r \in \mathbb{N}$.

i) If $K(x, y), L(x, y)$ are both $\mathrm{ETP}_{2}\left(\mathrm{ERR}_{2}\right)$ on $X \times Y$, then $K(x, y) L(x, y)$ is $\mathrm{ETP}_{2}\left(\mathrm{ERR}_{2}\right)$ on $X \times Y$.

ii) If $K(x, y)$ is $\mathrm{ETP}_{2}\left(\mathrm{ERR}_{2}\right)$ on $X \times Y$, then the kernel $L(x, y):=K(x, y)^{-1}$ is $\mathrm{ERR}_{2}\left(\mathrm{ETP}_{2}\right)$ on $X \times Y$.

iii) If $f \in C_{r-1}(X), g \in C_{r-1}(Y)$ are both positive on $X$ and $Y$ respectively and $K(x, y)$ is $\mathrm{ESR}_{r}$ on $X \times Y$, then the kernel

$$
L(x, y):=f(x) g(y) K(x, y), \quad(x, y) \in X \times Y,
$$

is $\mathrm{ESR}_{r}$ on $X \times Y$ with $\varepsilon_{m}(L)=\varepsilon_{m}(K), m=1, \ldots, r$.

iv) If $\phi: U \rightarrow X, \psi: V \rightarrow Y$ are both strictly monotone and surjective functions with inverses $\phi^{-1} \in C_{r-1}(X), \psi^{-1} \in C_{r-1}(Y)$ and $K(x, y)$ is $\operatorname{ESR}_{r}$ on $X \times Y$, then the kernel

$$
L(u, v):=K(\phi(u), \psi(v)), \quad(u, v) \in U \times V,
$$

is $\mathrm{ESR}_{r}$ on $\mathrm{U} \times \mathrm{V}$ with $\varepsilon_{m}(L)=\left(\operatorname{sign}\left(\phi^{\prime}(u) \psi^{\prime}(v)\right)\right)^{m(m-1) / 2} \varepsilon_{m}(K), m=1, \ldots, r$.

v) Suppose that $L(x, y)$ and $M(y, z)$ are both $\mathrm{ESR}_{r}$ on $X \times Y$ and $Y \times Z$ respectively. If $\rho$ is a positive $\sigma$-finite measure on $Y$ with at least $r$ points of increase and the kernel $K$ is defined by

$$
K(x, z):=\int_{Y} L(x, y) M(y, z) d \rho(y), \quad(x, y) \in X \times Z,
$$

the integral being absolutely convergent on $X \times Z$ such that $r$-fold differentiation with respect to $x$ and $z$ under the integral sign is permissable, then $K(x, z)$ is $\mathrm{ESR}_{r}$ on $X \times Z$ with $\varepsilon_{m}(K)=\varepsilon_{m}(L) \varepsilon_{m}(M), m=1, \ldots, r$. 
The following result deals with maxima of $\mathrm{ESR}_{2}$ function (Karlin (1968), pp. 158, 159).

LEMMA 1.4. Suppose that $X, Y \subset \mathbb{R}$ are open intervals and the real function $K(x, y)$ is $\mathrm{ETP}_{2}\left(\mathrm{ERR}_{2}\right)$ on $X \times Y$. Assume also that for each fixed $x \in X K(x, y)$ has a unique maximum at $y=\phi(x)$ which is a differentiable function of $x$. Then $\phi$ is a strictly increasing (decreasing) function of $x$.

Now we turn to sign regular properties of the noncentral densities in (1.2), (1.4), (1.7), (1.8). First we consider the dependence on the argument $x$ and the noncentrality parameter $\lambda$. Applying Lemma 1.3 repeatedly to the representations (1.12), (1.9), (1.15), (1.4) in Karlin (1968), pp. 118 - 121, the following result is established.

LEMMA 1.5. Suppose that $n, n_{1}, n_{2}, m>0$ are fixed. Then $p_{n, \lambda}(x)$ and $g_{n_{1}, n_{2}, \lambda}(x)$ are $\mathrm{ETP}_{\infty}$ with respect to $(x, \lambda)$ on $(0, \infty) \times(0, \infty)$ whereas $h_{m, \lambda}(x)$ is $\mathrm{ETP}_{\infty}$ with respect to $(x, \lambda)$ on $\mathbb{R} \times \mathbb{R}$.

Second we deal with the $\chi_{n}^{2}(\lambda)$ density regarded as a function of the argument $x$ only.

Lemma 1.6. Suppose that $n>0$ and $\lambda \geq 0$ are fixed. Then we have:

i) $p_{n, \lambda}(x-y)$ is $\mathrm{TP}_{\left\lfloor\frac{n}{2}\right\rfloor+1}$ with respect to $(x, y)$ on $\mathbb{R} \times \mathbb{R}$ (for the definition of the symbol $\lfloor\xi\rfloor$ see Section 0) and $p_{2, \lambda}(x)$ is $\mathrm{PF}_{2}$, i.e. $p_{2, \lambda}(x-y)$ is $\mathrm{TP}_{2}$ with respect to $(x, y)$ on $\mathbb{R} \times \mathbb{R}$,

ii) $p_{n, \lambda}(x+y)$ is $\operatorname{ERR}_{\left\lfloor\frac{n}{2}\right\rfloor+1}$ with respect to $(x, y)$ on $(0, \infty) \times(0, \infty)$ and moreover it is $\mathrm{ERR}_{\infty}$ when $n$ is an even integer,

iii) $p_{n, 0}(x y) \mathrm{ERR}_{\infty}$ with respect to $(x, y)$ on $(0, \infty) \times(0, \infty)$ whereas $p_{n, \lambda}(x y)$ is $\mathrm{ERR}_{2}$ on $(0, \infty) \times(0, \infty)$ if and only if $\lambda \leq n$.

Proof. i) The first part is established in Karlin (1968), pp. $107-109$, and the asserted $\mathrm{TP}_{2}$-property of $p_{2, \lambda}(x-y)$ is equivalent to the log-concavity of $p_{2, \lambda}(x)$ on $(0, \infty)$ which has been proved by Das Gupta, Sarkar (1984), pp. 56, 58. (See also Theorem 1.2 below where a different proof for the log-concavity of $p_{n, \lambda}(x)$ is given, $n \geq 2$.)

ii) Putting $q_{n, \lambda}(x):=e^{x / 2} p_{n, \lambda}(x)$ in (1.12) from Lemma 2.3 in Karlin (1968), p. 109 , we obtain that $q_{n, \lambda}(x+y)$ is $\operatorname{ERR}_{\infty}$ when $n$ is an even integer and in general the ERR $\left\lfloor\frac{n}{2}\right\rfloor+1$-property follows from the proof of Theorem 2.1 of Karlin (1968), pp. 107, 108. Via Lemma 1.3, iii) these results are transfered to $p_{n, \lambda}(x+y)$.

iii) Since the function $e^{-x y}$ is $\mathrm{ERR}_{\infty}$ the first assertion immediately follows from (1.1) and Lemma 1.3, iii). In order to prove the second one we observe that

$$
\operatorname{det}\left(\frac{\partial^{i+j-2}}{\partial x^{i-1} \partial y^{j-1}} p_{n, \lambda}(x y)\right)_{i, j=1}^{2}=p_{n, \lambda}(x y)^{2} \frac{\partial^{2}}{\partial x \partial y} \log p_{n, \lambda}(x y)
$$

Because of $p_{n, \lambda}(x y)>0$ for $x, y>0$, we have that $p_{n, \lambda}(x y)$ is $\operatorname{ERR}_{2}$ on $(0, \infty) \times(0, \infty)$ if and only if the right hand side of $(1.16)$ is negative on $(0, \infty) \times(0, \infty)$. By $(1.10)$ and (1.12) we get $\left(\nu=\frac{n-2}{2}\right)$

$$
p_{n, \lambda}(x y)=\frac{1}{2^{n / 2} \Gamma\left(\frac{n}{2}\right)} e^{-(\lambda+x y) / 2}(x y)^{\nu} \prod_{k=1}^{\infty}\left(1+\frac{\lambda x y}{j_{\nu, k}^{2}}\right)
$$

giving for $x, y>0$ (observe (1.11))

$$
\frac{\partial^{2}}{\partial x \partial y} \log p_{n, \lambda}(x y)=-\frac{1}{2}+\lambda \sum_{k=1}^{\infty} \frac{j_{\nu, k}^{2}}{\left(\lambda x y+j_{\nu, k}^{2}\right)^{2}}
$$


and

$$
\sup _{x, y,>0} \frac{\partial^{2}}{\partial x \partial y} \log p_{n, \lambda}(x y)=-\frac{1}{2}+\lambda \sum_{k=1}^{\infty} \frac{1}{j_{\nu, k}^{2}}=-\frac{1}{2}\left(1-\frac{\lambda}{n}\right) .
$$

Summarizing the proof is complete.

Next we have a look at the central $\chi_{n}^{2}$ density and the density of the central rescaled $\tilde{F}_{n_{1}, n_{2}}$ distribution both regarded as functions of $x$ and the degrees of freedom.

LEMMA 1.7.

i) The function $p_{n, 0}(x)$ is $\mathrm{ETP}_{\infty}$ with respect to $(x, n)$ on $(0, \infty) \times(0, \infty)$.

ii) If $n_{2}>0\left(n_{1}>0\right)$ is fixed, then $\tilde{g}_{n_{1}, n_{2}, 0}(x)$ is $\mathrm{ETP}_{\infty}\left(\mathrm{ERR}_{\infty}\right)$ with respect to $\left(x, n_{1}\right)\left(\left(x, n_{2}\right)\right)$ on $(0, \infty) \times(0, \infty)$.

Proof. i) This part follows readily by an application of Lemma 1.3, iii), iv) to (1.1), since the function $e^{x y}$ is $\operatorname{ETP}_{\infty}$.

ii) By Lemma 1.3, iv) it is easy to verify that the kernels

$$
K_{1}(x, y):=\left(\frac{x}{1+x}\right)^{y}, K_{2}(x, y):=\left(\frac{1}{1+x}\right)^{y}, x, y>0
$$

are $\mathrm{ETP}_{\infty}$ and $\mathrm{ERR}_{\infty}$ on $(0, \infty) \times(0, \infty)$ respectively. Now another application of Lemma 1.3, iii) to (1.5) proves our assertion. We remark that in Das Gupta, Perlman (1974) the $\mathrm{TP}_{2}$-property is shown.

A short inspection of the central $F_{n_{1}, n_{2}}$ density in (1.6) illustrates that $g_{n_{1}, n_{2}}(x)$ is neither $\mathrm{TP}_{2}$ nor $\mathrm{RR}_{2}$ with respect to $\left(x, n_{1}\right)$ or $\left(x, n_{2}\right)$. Also the central $t_{m}$ density in (1.3) fails to satisfy $\mathrm{TP}_{2}$ or $\mathrm{RR}_{2}$ with respect to $(x, m)$ on $\mathbb{R} \times(0, \infty)$.

LEMMA 1.8. Suppose that $\lambda>0$ is fixed. Then we have:

i) $p_{n, \lambda}(x)$ is $\mathrm{TP}_{2}$ with respect to $(x, n)$ on $(0, \infty) \times[2, \infty)$, fixed),

ii) $\tilde{g}_{n_{1}, n_{2}, \lambda}(x)$ is $\mathrm{ETP}_{2}$ with respect to $\left(x, n_{1}\right)$ on $(0, \infty) \times(0, \infty)\left(n_{2}>0\right.$ being fixed).

iii) $\tilde{g}_{n_{1}, n_{2}, \lambda}(x)$ is $\mathrm{ERR}_{2}$ with respect to $\left(x, n_{2}\right)$ on $(0, \infty) \times(0, \infty)\left(n_{1}>0\right.$ being

Proof. Part i) is established in Das Gupta, Sarkar (1984), p. 56, (see also Ghosh (1973)) and for ii) we rewrite (1.7) as

$$
\tilde{g}_{n_{1}, n_{2}, \lambda}(x)=\frac{1}{x}\left(\frac{x}{1+x}\right)^{n_{1} / 2}\left(\frac{1}{1+x}\right)^{n_{2} / 2} \int_{0}^{\infty} K\left(n_{1}, n_{2}, y\right)\left(\frac{x}{1+x}\right)^{y} d \rho(y)
$$

where $\rho$ is the measure attributing the Poisson weight $\frac{e^{-\lambda / 2}}{j !}\left(\frac{\lambda}{2}\right)^{j}$ to the point $y=$ $j ; j \in \mathbb{N}_{0}$, and the kernel $K\left(n_{1}, n_{2}, y\right)$ is defined by

$$
K\left(n_{1}, n_{2}, y\right):=\frac{\Gamma\left(\frac{n_{1}+n_{2}}{2}+y\right)}{\Gamma\left(\frac{n_{1}}{2}+y\right) \Gamma\left(\frac{n_{2}}{2}\right)} .
$$

On account of Euler's well-known integral for the Betafunction

$$
\frac{\Gamma\left(\frac{n_{1}}{2}+y\right) \Gamma\left(\frac{n_{2}}{2}\right)}{\Gamma\left(\frac{n_{1}+n_{2}}{2}+y\right)}=\int_{0}^{1} t^{\frac{n_{1}}{2}+y-1}(1-t)^{\frac{n_{2}}{2}-1} d t, \quad n_{1}, n_{2}>0, y \geq 0
$$


repeated applications of the composition rules in Lemma 1.3 and of (1.17) to (1.18) establish part ii). Very similar reasonings prove iii).

The above collected results for sign regularity of the noncentral densities can for instance be used to derive a couple of monotonicity properties of the power functions of various classical statistical tests and of percentage points (e.g. Ghosh (1973)). Also in some cases these properties of the densities can be transfered to the corresponding distribution functions (e.g. Das Gupta, Sarkar (1974), Finner, Roters (1993), Ghosh (1973)).

2. Bell-shape and unimodality. This section is devoted to our main results. To begin we consider the known results of Das Gupta, Sarkar (1984) and Minko, Petunin (1988) on the unimodality of the $\chi_{n}^{2}(\lambda)$ distribution.

Theorem 2.1. Suppose that $n \geq 2, \lambda \geq 0$.

i) The $\chi_{n}^{2}(\lambda)$ distribution is strongly unimodal. Moreover the function $\log p_{n, \lambda}$ is strictly concave on $(0, \infty)$ unless $(n, \lambda)=(2,0)$, where $p_{2,0}(x)=\frac{1}{2} e^{-x / 2}$.

ii) If $n>2, \lambda \geq 0$ or $n=2, \lambda>2$, then the $\chi_{n}^{2}(\lambda)$ distribution is strictly unimodal whereas $p_{2, \lambda}$ is strictly decreasing on $[0, \infty)$ when $\lambda \leq 2$.

Proof. We present a short reasoning different from the original one given by the above mentioned authors. As in the proof of Lemma 1.6, iii) we employ the representations (1.10) and (1.12) implying $\left(\nu=\frac{n-2}{2}\right)$

$$
p_{n, \lambda}(x)=\frac{1}{2^{n / 2} \Gamma\left(\frac{n}{2}\right)} e^{-(\lambda+x) / 2} x^{\nu} \prod_{k=1}^{\infty}\left(1+\frac{\lambda x}{j_{\nu, k}^{2}}\right), \quad x>0
$$

and

$$
\begin{gathered}
\frac{p_{n, \lambda}^{\prime}(x)}{p_{n, \lambda}(x)}=\frac{d}{d x} \log p_{n, \lambda}(x)=-\frac{1}{2}+\frac{\nu}{x}+\lambda \sum_{k=1}^{\infty} \frac{1}{\lambda x+j_{\nu, k}^{2}} \\
\left(\frac{d}{d x}\right)^{2} \log p_{n, \lambda}(x)=-\frac{\nu}{x^{2}}-\lambda^{2} \sum_{k=1}^{\infty} \frac{1}{\left(\lambda x+j_{\nu, k}^{2}\right)^{2}} .
\end{gathered}
$$

From (2.2) we infer the statement on the concavity and part i) follows from Ibragimov's criterion for strong unimodality. To establish ii) in view of (2.1) and (1.11) we observe

$$
\lim _{x \rightarrow 0+} \frac{d}{d x} \log p_{n, \lambda}(x)= \begin{cases}-\frac{1}{2}+\lambda \sum_{k=1}^{\infty} \frac{1}{j_{0, k}^{2}}=-\frac{1}{2}\left(1-\frac{\lambda}{2}\right), & n=2 \\ \infty & n>2\end{cases}
$$

and

$$
\lim _{x \rightarrow \infty} \frac{d}{d x} \log p_{n, \lambda}(x)=-\frac{1}{2}
$$

in any case. Further, by $(2.2)$, for $x>0$ we have $\left(\frac{d}{d x}\right)^{2} \log p_{n, \lambda}(x)<0$ unless $(n, \lambda)=(2,0)$ where $p_{2,0}(x)=\frac{1}{2} e^{-x / 2}$. Hence, if $n>2, \lambda \geq 0$ or $n=2, \lambda>2$, then the derivative $p_{n, \lambda}^{\prime}$ possesses exactly one zero on $(0, \infty)$ being a simple one. 
This implies the strict unimodality. Finally, if $n=2, \lambda \leq 2$, then (2.1) for $x>0$ yields (use (1.11) again)

$$
\frac{p_{2, \lambda}^{\prime}(x)}{p_{2, \lambda}(x)} \begin{cases}=-\frac{1}{2} & , \lambda=0 \\ <-\frac{1}{2}+\lambda \sum_{k=1}^{\infty} \frac{1}{j_{0, k}^{2}}=-\frac{1}{2}\left(1-\frac{\lambda}{2}\right) \leq 0 & , 0<\lambda \leq 2\end{cases}
$$

which completes the proof.

Theorem 2.2. If $n>2, \lambda \geq 0$, then the density $p_{n, \lambda}$ of the $\chi_{n}^{2}(\lambda)$ distribution is bell-shaped of order $\left\lfloor\frac{n}{2}\right\rfloor$.

Proof. For the central case $\lambda=0$ the reasoning has been given in Section 0 . Thus in the sequel we assume that $\lambda>0$. Further suppose that $k \in \mathbb{N}$ and $N_{k}$ denotes the number of zeros of $p_{n, \lambda}^{(k)}$ on $(0, \infty)$ counting multiplicities. We derive lower and upper bounds for $N_{k}$.

Let $k<\frac{n}{2}$. Then on account of the Rodrigues formula for Laguerre polynomials (0.2) and (1.2) we get

$$
p_{n, \lambda}^{(k)}(x)=\frac{k !}{2^{k}} \sum_{j=0}^{\infty} \frac{e^{-\lambda / 2}}{j !}\left(\frac{\lambda}{2}\right)^{j} p_{n+2 j, 0}(x)\left(\frac{x}{2}\right)^{-k} L_{k}^{\left(\frac{n}{2}+j-k-1\right)}\left(\frac{x}{2}\right), \quad x>0 .
$$

Next, by the orthogonality of the Laguerre polynomials (see Szegö (1985), p.100, and observe $\left.k<\frac{n}{2}\right)$ for $\nu=0, \ldots, k-1$ and all $j \in \mathbb{N}_{0}$ we obtain

$$
\begin{aligned}
& \int_{0}^{\infty} x^{\nu} p_{n+2 j, 0}(x)\left(\frac{x}{2}\right)^{-k} L_{k}^{\left(\frac{n}{2}+j-k-1\right)}\left(\frac{x}{2}\right) d x \\
= & \frac{2^{\nu}}{\Gamma\left(\frac{n}{2}+j\right)} \int_{0}^{\infty} e^{-\xi} \xi^{\frac{n}{2}+j-k-1} L_{k}^{\left(\frac{n}{2}+j-k-1\right)}(\xi) \xi^{\nu} d \xi=0
\end{aligned}
$$

and hence

$$
\int_{0}^{\infty} x^{\nu} p_{n, \lambda}^{(k)}(x) d x=0, \quad \nu=0, \ldots, k-1 .
$$

Thus $p_{n, \lambda}^{(k)}$ changes sign at least $k$ times on $(0, \infty)$ (see Polya, Szegö, vol.I (1971), p. 65) which implies $N_{k} \geq k$.

Concerning the upper estimate we employ (2.3) again together with the representations

$$
L_{k}^{(\alpha)}(x)=\sum_{\nu=0}^{k}\left(\begin{array}{l}
k+\alpha \\
k-\nu
\end{array}\right) \frac{(-x)^{\nu}}{\nu !}
$$

for the Laguerre polynomials (see Szegö (1985), p. 101, (5.1.6)) and

$$
\left(\begin{array}{l}
k+\alpha \\
k-\nu
\end{array}\right)=\frac{\Gamma(k+\alpha+1)}{(k-\nu) ! \Gamma(\alpha+\nu+1)}
$$

for the binomial. This gives

$$
p_{n, \lambda}^{(k)}(x)=\frac{e^{-(\lambda+x) / 2}}{2^{k+1}}\left(\frac{x}{2}\right)^{\frac{n}{2}-k-1} \sum_{j=0}^{\infty} \sum_{\nu=0}^{k}\left(\begin{array}{l}
k \\
\nu
\end{array}\right) \frac{(-1)^{\nu}}{j ! \Gamma\left(\frac{n}{2}+j+\nu-k\right)}\left(\frac{x}{2}\right)^{j+\nu}\left(\frac{\lambda}{2}\right)^{j}
$$


and further $(j+\nu=\rho)$

$$
p_{n, \lambda}^{(k)}(x)=\frac{e^{-(\lambda+x) / 2}}{2^{k+1}}\left(\frac{x}{2}\right)^{\frac{n}{2}-k-1} \sum_{\rho=0}^{\infty}\left(\frac{x}{2}\right)^{\rho}\left(\frac{\lambda}{2}\right)^{\rho} \frac{a_{k}(\rho)}{\Gamma\left(\frac{n}{2}+\rho-k\right) \Gamma(\rho+1)}
$$

where

$$
a_{k}(\rho)=\sum_{\nu=0}^{k}\left(\begin{array}{l}
k \\
\nu
\end{array}\right)(-1)^{\nu}\left(\frac{\lambda}{2}\right)^{-\nu} \rho(\rho-1) \ldots(\rho-\nu+1)
$$

is a real polynomial of $\rho$ with precise degree $k$. Thus the coefficients of the power series part in (2.5) change their signs at most $k$ times and by problem 38, p. 43 , of Polya, Szegö, vol. II, we get $N_{k} \leq k$. Summarizing the proof is complete.

In the sequel by $M=M(\lambda)$ we denote the unique mode if it exists. Occasionally we specify this mode by $M_{n}^{\chi^{2}}(\lambda), M_{n_{1}, n_{2}}^{F}(\lambda), M_{n_{1}, n_{2}}^{\tilde{F}}(\lambda), M_{m}^{t}(\lambda)$ for the distributions $\chi_{n}^{2}(\lambda), F_{n_{1}, n_{2}}(\lambda), \tilde{F}_{n_{1}, n_{2}}(\lambda), t_{m}(\lambda)$ respectively. Further we mention that the unimodality of the $\chi_{n}^{2}(\lambda)$ distribution as stated in Theorem 2.1, ii) also is a consequence of Theorem 2.2. This is obvious for $n>2, \lambda \geq 0$, and if $n=2$, then for $p_{2, \lambda}^{\prime}$ from the proof of Theorem 2.2 we conclude

$$
N_{1}=\left\{\begin{array}{lll}
0, & \text { if } \quad 0 \leq \lambda \leq 2 \\
1, & \text { if } \quad \lambda>2
\end{array}\right.
$$

here we have used the formulae $p_{2, \lambda}(0+)=\frac{1}{2} e^{-\lambda / 2}>0, p_{2, \lambda}^{\prime}(0+)=\frac{1}{4} e^{-\lambda / 2}\left(\frac{\lambda}{2}-1\right)$ which result from (1.2) and (2.3) respectively (note that $L_{1}^{(\alpha)}(x)=-x+\alpha+1$, Szegö (1985), p. 101). Consequently $\chi_{n}^{2}(\lambda)$ has a unique mode satisfying

$$
M_{n}^{\chi^{2}}(\lambda)\left\{\begin{array}{l}
>0, \text { if } n>2, \lambda \geq 0 \text { or } n=2, \lambda>2 \\
=0, \text { if } n=2,0 \leq \lambda \leq 2
\end{array}\right.
$$

THEOREM 2.3. If $n_{1}>2, n_{2}>0, \lambda \geq 0$, then the density $g_{n_{1}, n_{2}, \lambda}$ of the $F_{n_{1}, n_{2}}(\lambda)$ distribution is bell-shaped of order $\left\lfloor\frac{n_{1}}{2}\right\rfloor$.

Proof. Again as in the proof of Theorem 2.2 we estimate $N_{k}$ the number of zeros of $g_{n_{1}, n_{2}, \lambda}^{(k)}$ on $(0, \infty)$ counting multiplicities. To this end we employ representation (1.15) of Lemma 1.2 which implies

$$
g_{n_{1}, n_{2}, \lambda}^{(k)}(x)=\left(\frac{n_{1}}{n_{2}}\right)^{k+1} \int_{0}^{\infty} p_{n_{1}, \lambda}^{(k)}\left(\frac{n_{1}}{n_{2}} x y\right) p_{n_{2}, 0}(y) y^{k+1} d y, x>0 .
$$

First let $1 \leq k<\frac{n_{1}}{2}, k \in \mathbb{N}$. Then using Fubini's theorem we compute the moments

$$
\begin{aligned}
& \int_{0}^{\infty} x^{\nu} g_{n_{1}, n_{2}, \lambda}^{(k)}(x) d x \\
= & \left(\frac{n_{1}}{n_{2}}\right)^{k-\nu} \int_{0}^{\infty}\left(\int_{0}^{\infty}\left(\frac{n_{1}}{n_{2}}\right)^{\nu+1} x^{\nu} y^{\nu+1} p_{n_{1}, \lambda}^{(k)}\left(\frac{n_{1}}{n_{2}} x y\right) d x\right) p_{n_{2}, 0}(y) y^{k-\nu} d y
\end{aligned}
$$

for $\nu=0, \ldots, k-1$. Upon substituting $\xi=\frac{n_{1}}{n_{2}} x y$ in the inner integral from (2.4) we obtain

$$
\int_{0}^{\infty} x^{\nu} g_{n_{1}, n_{2}, \lambda}^{(k)}(x) d x=0, \quad \nu=0, \ldots, k-1
$$


Thus $g_{n_{1}, n_{2}, \lambda}^{(k)}$ changes sign at least $k$ times on $(0, \infty)$ and hence $N_{k} \geq k$. Concerning the upper estimate from (2.8) we get

$$
g_{n_{1}, n_{2}, \lambda}^{(k)}(x)=\left(\frac{n_{1}}{n_{2}}\right)^{k+1} \frac{1}{x^{k+2}} \int_{0}^{\infty} p_{n_{1}, \lambda}^{(k)}\left(\frac{n_{1}}{n_{2}} t\right) p_{n_{2}, 0}\left(\frac{t}{x}\right) t^{k+1} d t, x>0
$$

for any $k \in \mathbb{N}$. By Lemmata 1.6 , iii) and 1.3, iv) the kernel $p_{n_{2}, 0}(t / x)$ is $\operatorname{ETP}_{\infty}$ and in view of the preceeding theorem the function $p_{n_{1}, \lambda}^{(k)}$ has precisely $k$ changes of sign on $(0, \infty)$ provided that $k<\frac{n_{1}}{2}$. Now another application of Theorem 3.2 in Karlin (1968), p. 239, yields $N_{k} \leq k$ which completes the proof.

Corollary 2.4. If $n_{2}>0$ and $n_{1}>2, \lambda \geq 0$ or $n_{1}=2, \lambda>2$, then the $F_{n_{1}, n_{2}}(\lambda)$ distribution is strictly unimodal whereas $g_{2, n_{2}, \lambda}$ is strictly decreasing on $[0, \infty)$ when $\lambda \leq 2$. In any of these cases the unique mode exists and satisfies

$$
M_{n_{1}, n_{2}}^{F}(\lambda) \begin{cases}>0, & \text { if } n_{1}>2, \lambda \geq 0 \text { or } n_{1}=2, \lambda>2 \\ =0, & \text { if } n_{1}=2,0 \leq \lambda \leq 2\end{cases}
$$

Proof. This follows from Theorem 2.3 directly when $n_{1}>2$ and if $n_{1}=2$, then from (2.9) and (2.6) we conclude $0 \leq N_{1} \leq 1$. Further, by (2.8) and the remarks following (2.6), we have $g_{2, n_{2}, \lambda}(0+)=\frac{n_{1}}{4} e^{-\lambda / 2}>0$ and $g_{2, n_{2}, \lambda}^{\prime}(0+)=$ $\frac{1}{16 n_{2}} n_{1}^{2}\left(n_{1}+2\right) e^{-\lambda / 2}\left(\frac{\lambda}{2}-1\right)$. Consequently for $g_{2, n_{2}, \lambda}^{\prime}$ from the proof of Theorem 2.3 (see (2.9)) we infer

$$
N_{1}= \begin{cases}0, & \text { if } 0 \leq \lambda \leq 2 \\ 1, & \text { if } \lambda>2\end{cases}
$$

which completes the reasonings for the corollary.

We mention that the statements of Theorem 2.3 and Corollary 2.4 hold for the rescaled $\tilde{F}_{n_{1}, n_{2}}(\lambda)$ distribution as well. In particular, by (1.8),

$$
M_{n_{1}, n_{2}}^{\tilde{F}}(\lambda)=\frac{n_{1}}{n_{2}} M_{n_{1}, n_{2}}^{F}(\lambda)
$$

is valid.

TheOREM 2.5. For all $m>0$ and $\lambda \in \mathbb{R}$ the density $h_{m, \lambda}$ of the $t_{m}(\lambda)$ distribution is bell-shaped.

Proof. For $k \in \mathbb{N}$ we let $N_{k}^{+}, N_{k}^{-}, N_{k}^{0}$ be the numbers of zeros of $h_{m, \lambda}^{(k)}$ on the sets $(0, \infty),(-\infty, 0),\{0\}$ respectively, each counting multiplicities. Our starting point is the representation (1.4) giving

$$
h_{m, \lambda}^{(k)}(x)=c \int_{0}^{\infty} t^{\frac{m-1+k}{2}} e^{-\frac{t}{2}} H_{k}\left(x \sqrt{\frac{t}{2 m}}-\frac{\lambda}{\sqrt{2}}\right) \exp \left(-\left(x \sqrt{\frac{t}{2 m}}-\frac{\lambda}{\sqrt{2}}\right)^{2}\right) d t
$$

where $x>0$,

$$
c:=\frac{(-1)^{k}}{2^{\frac{m+k+1}{2}} \Gamma\left(\frac{m}{2}\right) m^{\frac{k+1}{2}} \sqrt{\pi}}
$$


and $H_{k}$ denotes the $k$-th Hermite polynomial (see (0.1)).

First we estimate the number of sign changes of $h_{m, \lambda}^{(k)}$ on $\mathbb{R}$ from below. Using Fubini's theorem and the substitution $\xi=x \sqrt{\frac{t}{2 m}}-\frac{\lambda}{\sqrt{2}}$ in (2.12) we compute the moments

$\int_{-\infty}^{\infty} x^{\nu} h_{m, \lambda}^{(k)}(x) d x=c(2 m)^{\frac{\nu+1}{2}} \int_{0}^{\infty} t^{\frac{n_{1}+k-\nu-2}{2}} e^{-\frac{t}{2}}\left(\int_{-\infty}^{\infty}\left(\xi+\frac{\lambda}{\sqrt{2}}\right)^{\nu} H_{k}(\xi) e^{-\xi^{2}} d \xi\right) d t$

for $\nu=0, \ldots, k-1$. Because of the orthogonality of the Hermite polynomials the inner integral vanishes. As before we conclude that $h_{m, \lambda}^{(k)}$ changes sign at least $k$ times on $\mathbb{R}$ and thus $N_{k}^{+}+N_{k}^{-}+N_{k}^{0} \geq k$.

Second we estimate each of the numbers $N_{k}^{+}, N_{k}^{-}, N_{k}^{0}$ from above.

i) Suppose that $x>0$ in (2.12). Upon substituting $y=x \sqrt{\frac{t}{2 m}}$ we get

$$
h_{m, \lambda}^{(k)}(x)=\frac{d}{x^{m+k+1}} \int_{0}^{\infty} e^{-\frac{m y^{2}}{x^{2}}} H_{k}\left(y-\frac{\lambda}{\sqrt{2}}\right) y^{m+k} e^{-\left(y-\frac{\lambda}{\sqrt{2}}\right)^{2}} d y
$$

where $d:=2 c(2 m)^{(m+k+1) / 2}$. Since the kernel $e^{-m y^{2} / x^{2}}$ is $\mathrm{ETP}_{\infty}$ again an application of Theorem 3.2 in Karlin (1968), p. 239, implies

$$
N_{k}^{+} \leq \text {number of sign changes of } H_{k}\left(y-\frac{\lambda}{\sqrt{2}}\right) \text { as } y \in(0, \infty) .
$$

ii) If $x<0$, then in (2.12) introduce the variable $y=-x \sqrt{\frac{t}{2 m}}$. Similarly we arrive at

$$
N_{k}^{-} \leq \text {number of sign changes of } H_{k}\left(-y-\frac{\lambda}{\sqrt{2}}\right) \text { as } y \in(0, \infty)
$$

iii) Finally, putting $x=0$ in (2.12) it is readily verified that

$$
h_{m, \lambda}^{(k)}(0)=\frac{(-1)^{k} \Gamma\left(\frac{m+k+1}{2}\right) e^{-\frac{\lambda^{2}}{2}}}{\Gamma\left(\frac{m}{2}\right) m^{\frac{m+1}{2}} \sqrt{\pi}} H_{k}\left(-\frac{\lambda}{\sqrt{2}}\right)
$$

In view of the well-known fact that $H_{k}$ has precisely $k$ real zeros all being simple ones, combining i), ii), iii), we end with $N_{k}^{+}+N_{k}^{-}+N_{k}^{0} \leq k$. This completes the proof.

If we pay attention to the case $k=1$ in the preceeding proof and observe that $H_{1}(y)=2 y$ (see Szegö (1985), p. 106), then we may exhibit the following important consequence.

COROLlaRY 2.6. For all $m>0$ and $\lambda \in \mathbb{R}$ the $t_{m}(\lambda)$ distribution is strictly unimodal and its mode satisfies

$$
\operatorname{sign} M_{m}^{t}(\lambda)=\operatorname{sign} \lambda, \quad M_{m}^{t}(-\lambda)=-M_{m}^{t}(\lambda) .
$$


3. Monotonicity properties. Plots of the noncentral densities suggest monotonic dependence of various quantities with respect to the noncentrality parameter and the degrees of freedom (e.g. Johnson et al. (1995), Kühlmeyer (1970), Narula, Levy (1975)). It is the aim of this section to establish proofs for some of these indicated properties. We start with a collection of some formulae which are readily derived from (1.1), (1.2), (1.5), (1.7).

LEMMA 3.1.

i) If $n, n_{1}>2, n_{2}>0, \lambda \geq 0$, then for $x>0$ we have

$$
\begin{gathered}
p_{n, \lambda}^{\prime}(x)=\frac{1}{2}\left(p_{n-2, \lambda}(x)-p_{n, \lambda}(x)\right), \\
\tilde{g}_{n_{1}, n_{2}, \lambda}^{\prime}(x)=\frac{n_{2}}{2}\left(\tilde{g}_{n_{1}-2, n_{2}+2, \lambda}(x)-\tilde{g}_{n_{1}, n_{2}+2, \lambda}(x)\right) .
\end{gathered}
$$

ii) If $n, n_{1}, n_{2}>0, \lambda \geq 0$, then for $x>0$ we have

$$
\begin{gathered}
\frac{\partial}{\partial \lambda} p_{n, \lambda}(x)=\frac{1}{2}\left(p_{n+2, \lambda}(x)-p_{n, \lambda}(x)\right), \\
\frac{\partial}{\partial \lambda} \tilde{g}_{n_{1}, n_{2}, \lambda}(x)=\frac{1}{2}\left(\tilde{g}_{n_{1}+2, n_{2}, \lambda}(x)-\tilde{g}_{n_{1}, n_{2}, \lambda}(x)\right) .
\end{gathered}
$$

iii) If $n, n_{1}>2, n_{2}>0, \lambda \geq 0$, then for $x>0$ we have

$$
\begin{gathered}
\frac{\partial}{\partial x} p_{n, \lambda}(x)=-\frac{\partial}{\partial \lambda} p_{n-2, \lambda}(x), \\
\frac{\partial}{\partial x} \tilde{g}_{n_{1}, n_{2}, \lambda}(x)=-n_{2} \frac{\partial}{\partial \lambda} \tilde{g}_{n_{1}-2, n_{2}+2, \lambda}(x) .
\end{gathered}
$$

We mention that formulae (3.1), (3.3) are contained in Johnson et al. (1995), pp. 442, 443, Cohen (1988), Ruben (1974). In view of (3.1), (3.2) we observe that the modes $M_{n}^{\chi^{2}}(\lambda), M_{n_{1}, n_{2}}^{\tilde{F}}(\lambda)$ occur as the unique solutions of the equations $p_{n-2, \lambda}(x)=p_{n, \lambda}(x)$ and $\tilde{g}_{n_{1}-2, n_{2}+2, \lambda}(x)=\tilde{g}_{n_{1}, n_{2}+2, \lambda}(x)$ respectively. This fact is already mentioned by Johnson et.al. (1995), p. 451. Next we turn to monotonicity properties of the modes with respect to the noncentrality parameter thereby extending the information obtained in (2.7), (2.10), (2.13).

Theorem 3.2

i) If $n>2(n=2)$ is fixed, then $M_{n}^{\chi^{2}}(\lambda)$ is a strictly increasing function of $\lambda \in[0, \infty)(\lambda \in[2, \infty))$.

ii) If $n_{1}>2, n_{2}>0\left(n_{1}=2, n_{2}>0\right)$ are fixed, then $M_{n_{1}, n_{2}}^{F}(\lambda)$ is a strictly increasing function of $\lambda \in[0, \infty)(\lambda \in[2, \infty))$.

iii) If $m>0$ is fixed, then $M_{m}^{t}(\lambda)$ is a strictly increasing function of $\lambda \in \mathbb{R}$.

Proof. Suppose that $p(x, \lambda)$ is any of the three noncentral densities and assume in the cases i) and ii) that $\lambda$ varies in the open intervals $(0, \infty)$ and $(2, \infty)$. By the results of the preceeding section $p$ attains a single maximum at $x=M(\lambda)$, the mode, which is the unique solution of the equation $\frac{\partial}{\partial x} p(x, \lambda)=0$. Since $\frac{\partial^{2}}{\partial x^{2}} p(M(\lambda), \lambda) \neq 0$, 
the implicit function theorem implies that $M(\lambda)$ is a differentiable function of $\lambda$. Now according to Lemma 1.5 for all cases $p(x, \lambda)$ is $\mathrm{ETP}_{\infty}$ and Lemma 1.4 proves the asserted monotonicity property with respect to $\lambda$ on the corresponding open intervals. The extension for part i), ii) to the boundary points $\lambda=0$ and $\lambda=2$ now follows from a simple continuity argument.

REMARK. We add a short direct proof of Theorem 3.2 , i) without refering to the theory of total positivity. To this end write $M(\lambda)=M_{n}^{\chi^{2}}(\lambda)$ as above. From $p_{n, \lambda}^{\prime}(M(\lambda))=0, \lambda>0$, and the implicit function theorem we get

$$
\frac{\partial^{2}}{\partial x^{2}} p_{n, \lambda}(M(\lambda)) \frac{d}{d \lambda} M(\lambda)+\frac{\partial}{\partial \lambda} \frac{\partial}{\partial x} p_{n, \lambda}(M(\lambda))=0 .
$$

Since $\frac{\partial^{2}}{\partial x^{2}} p_{n, \lambda}(M(\lambda))<0$ and $\frac{\partial}{\partial x} \frac{\partial}{\partial \lambda} p_{n, \lambda}(M(\lambda))=\frac{1}{2} p_{n+2, \lambda}^{\prime}(M(\lambda)) \quad($ see (3.3)), we show that the latter quantity is positive. This follows from (1.13) which implies

$$
p_{n+2, \lambda}^{\prime}(M(\lambda))=\int_{0}^{M(\lambda)} p_{2,0}(y) p_{n, \lambda}^{\prime}(M(\lambda)-y) d y>0 .
$$

Next we turn to monotonicity properties for the mode of the $\chi_{n}^{2}(\lambda)$ and $\tilde{F}_{n_{1}, n_{2}}(\lambda)$ distribution with respect to the degrees of freedom. In the central case from (1.1), (1.5) it is easily verified that

$$
M_{n}^{\chi^{2}}(0)=n-2, M_{n_{1}, n_{2}}^{\tilde{F}}(0)=\frac{n_{1}-2}{n_{2}+2}, \quad n, n_{1} \geq 2, n_{2}>0
$$

from which the monotonic dependence is obvious. For the noncentral mode $M_{n}^{\chi^{2}}(\lambda)$ Sen $(1989$, p. $109,(2.11))$ proved $M_{n-2}^{\chi^{2}}(\lambda) \leq M_{n}^{\chi^{2}}(\lambda)$ provided $\lambda \geq 0, n>2$. The general case is treated in

TheOREM 3.3. Suppose that $\lambda \geq 0$ is fixed.

i) Then $M_{n}^{\chi^{2}}(\lambda)$ is an increasing function of $n \in[2, \infty)$.

ii) If $n_{2}>0$ is fixed, then $M_{n_{1}, n_{2}}^{\tilde{F}}(\lambda)$ is a strictly increasing function of $n_{1} \in(2, \infty)$ whereas for fixed $n_{1}>2 M_{n_{1}, n_{2}}^{\tilde{F}}(\lambda)$ is a strictly decreasing function of $n_{2} \in(0, \infty)$.

Proof. i) Suppose that $2 \leq \nu_{1}<\nu_{2}, 0<x_{1}<x_{2}$. Then, by the $\mathrm{TP}_{2}$-property of $p_{n, \lambda}(x)$ according to Lemma $\left.1.8, \mathrm{i}\right)$,

$$
\operatorname{det}\left(p_{\nu_{i}, \lambda}\left(x_{j}\right)\right)_{i, j=1}^{2} \geq 0
$$

holds which implies that the quotient

$$
\frac{p_{\nu_{2}, \lambda}(x)}{p_{\nu_{1}, \lambda}(x)} \text { is increasing for } x \in(0, \infty) .
$$

Let $M_{i}:=M_{\nu_{i}}^{\chi^{2}}(\lambda), i=1,2$, and assume that $M_{2}<M_{1}$. Because $p_{\nu_{1}, \lambda}\left(p_{\nu_{2}, \lambda}\right)$ is a strictly increasing (decreasing) function on $\left(M_{2}, M_{1}\right)$ we have that $p_{\nu_{2}, \lambda}(x) / p_{\nu_{1}, \lambda}(x)$ is strictly decreasing for $x \in\left(M_{2}, M_{1}\right)$. This contradicts (3.7).

ii) The central case is obvious and if $\lambda>0$, then both statements follow from an application of Lemma 1.4 in connection with Lemma 1.8, ii), iii). 
Concerning the mode $M_{n_{1}, n_{2}}^{\tilde{F}}(\lambda)$ we exhibit that the results of Theorem 3.3, ii) cannot be transfered to the mode of $F_{n_{1}, n_{2}}(\lambda)$, since $M_{n_{1}, n_{2}}^{F}(\lambda)=\frac{n_{2}}{n_{1}} M_{n_{1}, n_{2}}^{\tilde{F}}(\lambda)$ (see (2.11)). Actually numerical calculations indicate that $M_{n_{1}, n_{2}}^{F}(\lambda)$ is monotonic with respect to the degress of freedom only when $\lambda$ is restricted to certain subintervals of $(0, \infty)$.

Supplementing Theorem 3.2 we prove

Theorem 3.4 Suppose that $n, n_{1}, n_{2}>2, m>0$ are fixed. Then

i) $p_{n, \lambda}\left(M_{n}^{\chi^{2}}(\lambda)\right)$ is a strictly decreasing function of $\lambda \in(0, \infty)$,

ii) $g_{n_{1}, n_{2}, \lambda}\left(M_{n_{1}, n_{2}}^{F}(\lambda)\right)$ is a strictly decreasing function of $\lambda \in(0, \infty)$,

iii) $h_{m, \lambda}\left(M_{m}^{t}(\lambda)\right)$ is a strictly increasing (decreasing) function of $\lambda \in(-\infty, 0)$ $(\lambda \in(0, \infty))$.

Proof. Assume that $p(x, \lambda)$ is any of the three noncentral densities with the corresponding mode $M(\lambda)$. In view of

$$
\frac{d}{d \lambda} p(M(\lambda), \lambda)=p^{\prime}(M(\lambda), \lambda) \frac{d}{d \lambda} M(\lambda)+\frac{\partial}{\partial \lambda} p(M(\lambda), \lambda)=\frac{\partial}{\partial \lambda} p(M(\lambda), \lambda)
$$

we determine the sign of the right most quantity.

i) In the $\chi_{n}^{2}(\lambda)$ case we employ (3.3) together with (1.13) giving

$$
\begin{aligned}
\frac{\partial}{\partial \lambda} p_{n, \lambda}(M(\lambda)) & =\frac{1}{2}\left(p_{n+2, \lambda}(M(\lambda))-p_{n, \lambda}(M(\lambda))\right) \\
& =\frac{1}{2}\left(\int_{0}^{\infty} p_{2,0}(y) p_{n, \lambda}(M(\lambda)-y) d y-p_{n, \lambda}(M(\lambda))\right) \\
& =\frac{1}{2} \int_{0}^{\infty} p_{2,0}(y)\left(p_{n, \lambda}(M(\lambda)-y)-p_{n, \lambda}(M(\lambda))\right) d y
\end{aligned}
$$

and the latter quantity obviously is negative.

ii) First we consider the $\tilde{F}_{n_{1}, n_{2}}(\lambda)$ distribution the mode of which satisfies

$$
M_{n_{1}, n_{2}}^{\tilde{F}}(\lambda)<M_{n_{1}+2, n_{2}-2}^{\tilde{F}}(\lambda)
$$

according to Theorem 3.3, ii). Using this information together with formula (3.6) of Lemma 3.1 , iii) we obtain

$$
\frac{\partial}{\partial \lambda} \tilde{g}_{n_{1}, n_{2}, \lambda}\left(M_{n_{1}, n_{2}}^{\tilde{F}}(\lambda)\right)=-\frac{1}{n_{2}-2} \frac{\partial}{\partial x} \tilde{g}_{n_{1}+2, n_{2}-2, \lambda}\left(M_{n_{1}, n_{2}}^{\tilde{F}}(\lambda)\right)<0
$$

which on account of (1.8), (2.11) implies

$$
\frac{\partial}{\partial \lambda} g_{n_{1}, n_{2}, \lambda}\left(M_{n_{1}, n_{2}}^{F}(\lambda)\right)<0 .
$$

iii) In the $t_{m}(\lambda)$ case differentiate (1.4) to obtain

$\frac{\partial}{\partial \lambda} h_{m, \lambda}(M(\lambda))=a \int_{0}^{\infty} t^{\frac{m-1}{2}} e^{-\frac{t}{2}} \exp \left(-\frac{1}{2}\left(M(\lambda) \sqrt{\frac{t}{m}}-\lambda\right)^{2}\right)\left(M(\lambda) \sqrt{\frac{t}{m}}-\lambda\right) d t$

where

$$
a:=\frac{1}{2^{\frac{m+1}{2}} \Gamma\left(\frac{m}{2}\right) \sqrt{m \pi}}
$$


Adding $c h_{m, \lambda}^{\prime}(M(\lambda))=0$ with a constant $c$ the choice of which is made below we get

$$
\begin{aligned}
\frac{\partial}{\partial \lambda} h_{m, \lambda}(M(\lambda)) & =a \int_{0}^{\infty} t^{\frac{m-1}{2}} e^{-\frac{t}{2}} \exp \left(-\frac{1}{2}\left(M(\lambda) \sqrt{\frac{t}{m}}-\lambda\right)^{2}\right) \\
& \times\left(M(\lambda) \sqrt{\frac{t}{m}}-\lambda\right)\left(1-c \sqrt{\frac{t}{m}}\right) d t .
\end{aligned}
$$

Now, putting $c:=M(\lambda) / \lambda, \lambda \neq 0$, we arrive at

$$
\operatorname{sign} \frac{\partial}{\partial \lambda} h_{m, \lambda}(M(\lambda))=-\operatorname{sign} \lambda, \quad \lambda \neq 0 .
$$

This completes the proof.

We close this section by investigating the variation of the $\chi_{n}^{2}(\lambda)$ and the $F_{n_{1}, n_{2}}(\lambda)$ density with respect to the noncentrality parameter.

Theorem 3.5. Suppose that $n, n_{1}, m, x>0, n_{2}>2$ are fixed. Then for all $\lambda>0$

$$
\frac{\partial}{\partial \lambda} p_{n, \lambda}(x)\left\{\begin{array}{c}
< \\
>
\end{array}\right\} 0 \quad \text { if } \quad x\left\{\begin{array}{c}
< \\
>
\end{array}\right\} M_{n+2}^{\chi^{2}}(\lambda)
$$

and

$$
\frac{\partial}{\partial \lambda} g_{n_{1}, n_{2}, \lambda}(x)\left\{\begin{array}{c}
< \\
>
\end{array}\right\} 0 \quad \text { if } \quad x\left\{\begin{array}{c}
< \\
>
\end{array}\right\} \frac{n_{2}\left(n_{1}+2\right)}{n_{1}\left(n_{2}-2\right)} M_{n_{1}+2, n_{2}-2}^{F}(\lambda) .
$$

Proof. The assertion concerning the $\chi_{n}^{2}(\lambda)$ case follows from (3.5) and Theorem 2.2. To prove the second part first we consider the rescaled $\tilde{F}_{n_{1}, n_{2}}(\lambda)$ distribution and write (3.6) in the form

$$
\frac{\partial}{\partial x} \tilde{g}_{n_{1}+2, n_{2}-2, \lambda}(x)=-\left(n_{2}-2\right) \frac{\partial}{\partial \lambda} \tilde{g}_{n_{1}, n_{2}, \lambda}(x) .
$$

Thus from Theorem 2.3 we conclude

$$
\frac{\partial}{\partial \lambda} \tilde{g}_{n_{1}, n_{2}, \lambda}(x)\left\{\begin{array}{c}
< \\
>
\end{array}\right\} 0 \quad \text { if } \quad x\left\{\begin{array}{c}
< \\
>
\end{array}\right\} M_{n_{1}+2, n_{2}-2}^{\tilde{F}}(\lambda)
$$

and (1.8), (2.11) finish the proof. $\square$

\section{REFERENCES}

[1] J. P. CoHen, Noncentral chi-square: Some observations and recurrence, Amer. Stat., 42 (1988), pp. 120-122.

[2] S. Das Gupta And M. D. Perlman, Power of the noncentral F-Test: Effect of additional variates on Hotelling's $T^{2}$-Test, J. Amer. Statist. Assoc., 69 (1974), pp. 174-180.

[3] S. DAS Dupta AND S. K. SARKAR, On $\mathrm{TP}_{2}$ and log-concavity, in Inequalities in Statistics and Probability, Y.L. Tong, ed., Lecture Notes-monograph series, Institute of Mathematical Statistics, vol. 5, 1984, pp. 54-58.

[4] S. Dharmadhikari And J. Joag-Dev, Unimodality, Convexity, and Applications, Academic Press, San Diego, 1988.

[5] H. Finner ANd M. Roters, Distribution functions and log-concavity, Comm. Statist. Theory Methods, 22 (1993), pp. 2381-2396. 
[6] B. K. Gноsн, Some monotonicity theorems for $\chi^{2}, F$ and $t$ distributions with applications, J. Royal Stat. Soc., Ser. B, 35 (1973), pp. 480-492.

[7] I. A. Ibragimov, On the composition of unimodal distributions, Theory Probab. Appl., 1 (1956), pp. 255-260.

[8] N. L. Johnson, S. Kotz, and S. Balakrishnan, Continuous Univariate Distributions, vol. II, Wiley, New York, 1995.

[9] S. Karlin, Total Positivity, vol. I, Stanford University Press, Stanford CA, 1968.

[10] M. KüHLMEYeR, Die Nichtzentrale $t$-Verteilung, Lecture Notes in Operations Research and Mathematical Systems, Springer, New York, 1970.

[11] A. A. Minko AND Y. I. Petunin, Unimodality of a noncentral $\chi^{2}$-distribution, Theory Probab. Math. Stat., 36 (1988), pp. 91-96.

[12] S. C. NARUla AND K. J. LeVy, Probability density plots of the noncentral $\chi^{2}$ and noncentral F distributions, Internat. Statist. Rev., 43 (1975), pp. 79-82.

[13] G. Polya And G. Szegö, Aufgaben und Lehrsätze aus der Analysis, Bd. I, II, Springer, Berlin, 1971.

[14] H. RuBEN, Non-central chi-square and gamma revisited, Comm. Statist. Theory Methods, 3 (1974), pp. 607-633.

[15] S. K. SARKAR, Some results of $\chi^{2}$ and a related distribution, Sankhyã, Ser. A, 45 (1983), pp. $253-255$.

[16] K. SATo AND M. YAmazato, On distribution functions of class $L$, Z. Wahrscheinlichkeitstheorie verw. Gebiete, 43 (1978), pp. 273-308.

[17] P. K. SEN, The mean-median-mode inequality and noncentral chi square distributions, Sankhyã, Ser. A, 51 (1989), pp. 106-114.

[18] G. Szegö, Orthogonal Polynomials, Amer. Math. Soc. Colloqu. Publ. 23, Providence, R. I., 1985.

[19] G. N. Watson, A Treatise on the Theory of Bessel Functions, Cambridge University Press, London, 1966.

[20] M. Yamazato, Unimodality of infinitely divisible distribution functions of class L, Ann. Probab., 6 (1978), pp. 523-531. 
